\title{
Modélisation et simulation numérique d'une chambre de postcombustion
}

\author{
Hatem Gacem $^{1, a}$, Mohamed Jemmali ${ }^{2}$ et Jamel Bessrour ${ }^{2}$ \\ ${ }^{1}$ Laboratoire d'Ingénierie des Systèmes Mécaniques et des Matériaux (LISMMA), Institut Supérieur de Mécanique de Paris \\ (SUPMECA), 3 rue Fernand Hainaut, 93407 Saint-Ouen Cedex, France \\ ${ }^{2}$ U.R. Mécanique appliquée, ingénierie et industrialisation, École Nationale d'Ingénieurs de Tunis (ENIT), BP 37, Le Belvédère, \\ 1002 Tunis, Tunisie
}

Reçu le 21 janvier 2004, accepté le 22 juin 2006

Résumé - Cette étude s'inscrit dans le cadre d'une approche globale d'unité d'incinération (four tournant, chambre de postcombustion, traitement des fumées), dont l'objectif est l'identification du comportement aéro-thermo-chimique de l'écoulement réactif au sein de la chambre de postcombustion. Le travail développé ici, dans une démarche d'ingénierie, s'intéresse tout particulièrement à la vérification de la règle des trois $\mathrm{T}$ (Température, Turbulence et Temps de séjours) dans l'unité primaire de traitement des fumées. L'étude est menée au travers de simulations numériques en écoulement réactif d'une chambre de postcombustion d'une unité fonctionnant en four tournant de 1,5 MW et consommant un combustible gazeux. Les simulations sont effectuées au moyen du code de calcul Phoenics, en utilisant le modèle de combustion turbulente Eddy Break Up couplé avec le modèle de turbulence $K-\varepsilon$.

Mots clés : Incinération / postcombustion / modélisation / simulation numérique / écoulement réactif / turbulence

\begin{abstract}
Flow modelling and numerical simulation of post combustion room. This study takes place in an engineering global study of incineration process (rotary kiln, post-combustion room, smoke treatment), which has as objective the identification of the aero-thermal and chemical behaviour of the reactive flow in the post combustion room. The work developed here, in an engineering approach, focuses in particular on the verification of the three $\mathrm{T}$ rule (Temperature, Turbulence and Time stay of gases) in the first treatment smoke unity. The study is done through numerical simulation in reactive flow of a post-combustion room. The incineration unity works with a rotary kiln of $1.5 \mathrm{MW}$ and consuming gas fuel. Phoenics is used to achieve simulation, taking into account turbulence and combustion interaction through the Eddy Break Up model coupled with the turbulence model $K-\varepsilon$.
\end{abstract}

Key words: Incineration / post combustion / modelling / numerical simulation / reactive flow / turbulence

\section{Introduction}

Les émissions polluantes issues de l'incinération des déchets solides sont liées aux dispositions géométriques de l'installation, au réglage des différents paramètres de la combustion et au type de déchets. Par rapport à un déchet donné, l'optimisation de la combustion est délicate à cause du nombre important de contraintes intervenant dans la mise au point d'une telle installation. La recherche des meilleurs paramètres pour avoir des conditions d'incinération optimales ne peut pas dispenser de

${ }^{a}$ Auteur pour correspondance : gacem.hatem@supmeca.fr l'installation d'unités de traitement des fumées pour le respect des normes en vigueur.

Dans le cas de l'incinération des déchets septiques hospitaliers, il est nécessaire, pour satisfaire aux normes relatives aux émissions et aux directives de la CEE/1999, d'introduire, à la sortie du four, des dispositions pour l'élimination des poussières, des émanations gazeuses, des métaux lourds et des polluants organiques (dioxines,... ). À ces fins, nous avons adopté une unité primaire de traitement des fumées par post-combustion suivie par un refroidissement des gaz (par injection d'air ou par récupération) et un cyclonage pour la séparation des poussières [1]. Une 


\section{Nomenclature}

\begin{tabular}{|c|c|c|}
\hline$C_{n}$ & variable d'avancement de la réaction du constituant $n$ & \\
\hline$C_{\mathrm{p}}$ & chaleur massique & $\mathrm{J} \cdot \mathrm{kg}^{-1} \cdot \mathrm{K}^{-1}$ \\
\hline$C_{\mathrm{EBU}}$ & constante du modèle Eddy Break & \\
\hline$C_{\varepsilon 1}$ & constante du modèle $K-\varepsilon$ & \\
\hline$C_{\varepsilon 2}$ & constante du modèle $K-\varepsilon$ & \\
\hline$d$ & diffusivité thermique & $\mathrm{m}^{2} \cdot \mathrm{s}^{-1}$ \\
\hline$d_{\mathrm{t}}$ & diffusivité turbulente & $\mathrm{m}^{2} \cdot \mathrm{s}^{-1}$ \\
\hline$\vec{f}$ & densité des forces volumiques & N.m ${ }^{-3}$ \\
\hline$h$ & enthalpie massique & ${\mathrm{J} . \mathrm{kg}^{-1}}^{-1}$ \\
\hline$j$ & vecteur de diffusion massique & $\mathrm{kg} \cdot \mathrm{m}^{-2} \cdot \mathrm{s}^{-1}$ \\
\hline$K$ & énergie cinétique turbulente & $\mathrm{m}^{2} \cdot \mathrm{s}^{-2}$ \\
\hline$l_{\mathrm{m}}$ & longueur de mélange & $\mathrm{m}$ \\
\hline$n$ & normale & \\
\hline$P$ & pression & $\mathrm{Pa}$ \\
\hline$R$ & constante massique du gaz parfait & $\mathrm{J} \cdot \mathrm{kg}^{-1} \cdot \mathrm{K}^{-1}$ \\
\hline$r$ & source de chaleur volumique & $\mathrm{W} \cdot \mathrm{m}^{-3}$ \\
\hline$T$ & température & $\mathrm{K}$ \\
\hline$V$ & vecteur vitesse & $\mathrm{m} \cdot \mathrm{s}^{-1}$ \\
\hline$Y_{n}$ & fraction massique du constituant $n$ & \\
\hline \multicolumn{3}{|c|}{ Symboles grecs } \\
\hline$\varepsilon$ & taux de dissipation de l'énergie cinétique turbulente & $\mathrm{m}^{2} \cdot \mathrm{s}^{-3}$ \\
\hline$\lambda$ & conductivité thermique & $\mathrm{W} \cdot \mathrm{m}^{-1} \cdot \mathrm{K}^{-1}$ \\
\hline$\mu$ & viscosité dynamique & $\mathrm{kg} \cdot \mathrm{m}^{-1} \cdot \mathrm{s}^{-1}$ \\
\hline$\mu_{\mathrm{t}}$ & viscosité dynamique turbulente & $\mathrm{kg} \cdot \mathrm{m}^{-1} \cdot \mathrm{s}^{-1}$ \\
\hline$\nu$ & viscosité cinématique & $\mathrm{m}^{2} \cdot \mathrm{s}^{-1}$ \\
\hline$\nu_{\mathrm{t}}$ & viscosité cinématique turbulente & $\mathrm{m}^{2} \cdot \mathrm{s}^{-1}$ \\
\hline$\rho$ & densité massique & $\mathrm{kg} \cdot \mathrm{m}^{-3}$ \\
\hline$\omega$ & taux moyen de réaction chimique & $\mathrm{s}^{-1}$ \\
\hline$\sigma_{\varepsilon}$ & constante du modèle $K-\varepsilon$ & \\
\hline \multicolumn{3}{|c|}{ indices et notations } \\
\hline $\mathrm{a}$ & air & \\
\hline $\mathrm{b}$ & brûleur & \\
\hline $\mathrm{c}$ & combustible & \\
\hline f & fumées & \\
\hline$i, j$ & composantes des vecteurs vitesses & \\
\hline $\bar{x}$ & moyenne classique d'une variable $x$ & \\
\hline$x^{\prime}$ & valeur fluctuante de $x: x=\bar{x}+x^{\prime}$ & \\
\hline$\tilde{x}$ & moyenne de Favre de $x$ & \\
\hline & valeur fluctuante de $x: x=\tilde{x}+x^{\prime \prime}$ & \\
\hline & $b_{i}=\sum a_{i} b_{i}$ & \\
\hline
\end{tabular}

installation supplémentaire de filtres textiles à manches combinées à certains additifs est également prévue.

L'objectif de cette étude est l'identification du comportement aérothermique de la chambre de postcombustion située en aval du four tunnel (Fig. 1). Il s'agit notamment de vérifier qu'elle satisfait à la réglementation de la CEE qui fixe les conditions de postcombustion par le respect de la règle des trois "T" (temps de séjours des gaz, température et turbulence). Ce critère exige une température supérieure ou égale à $750{ }^{\circ} \mathrm{C}$, un temps de séjour des gaz minimal de deux secondes et un écoulement turbulent ce qui permettrait une importante réduction des gaz nocifs et malodorants. À cet effet, l'utilisation de la MFN (Mécanique des Fluides Numérique) et de la simulation, pour répondre à la complexité des phénomènes physiques mis en jeu est devenue importante en ingénierie notamment devant l'impossibilité de disposer de relevés expérimentaux au stade de l'avant-projet d'une installation industrielle [2-4]. Elle permet, en particulier, d'étudier l'influence de la conception de l'installation et du réglage de certains paramètres sur les caractéristiques de l'incinération.

Dans une première étape de l'étude, on a procédé à la modélisation de l'écoulement réactif par le choix de modèles de turbulence et de combustion et l'établissement des lois de conservation mettant en jeu les variables d'état caractérisant l'écoulement, à savoir les vitesses, pression, température, densité, grandeurs turbulentes et espèces réactives. Les calculs sont effectués à l'aide du code Phoenics [5]. 


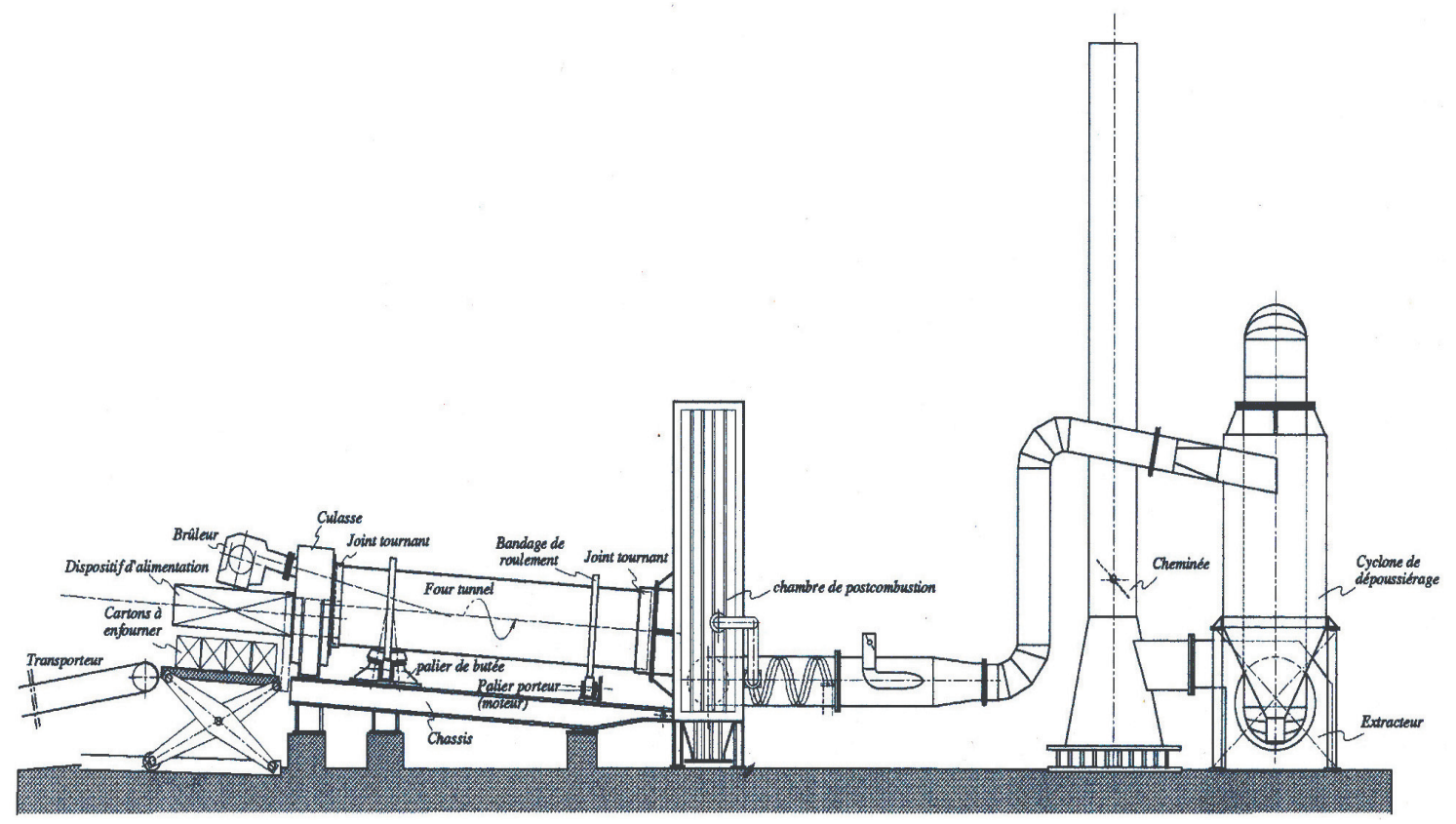

Fig. 1. Schéma de l'installation.

En second lieu, on a présenté les résultats des simulations numériques, de l'écoulement réactif, dans la chambre de postcombustion qui ont permis de prévoir les différentes situations d'écoulement et de discuter la validité de la conception adoptée. On a étudié notamment l'influence de la variation de deux paramètres : la hauteur du muret de séparation des deux compartiments de la chambre et l'effet de la disposition et du nombre de gicleurs d'air neuf.

\section{Géométrie}

La chambre de postcombustion étudiée est l'unité primaire de traitement des fumées d'une installation d'incinération de déchets septiques hospitaliers [1] (Fig. 1).

C'est une enceinte parallélépipédique conçue autour d'une ossature, d'un blindage en acier et d'un garnissage réfractaire. Un muret en maçonnerie réfractaire subdivise la chambre en deux compartiments identiques afin d'assurer, par un double parcours des fumées, le temps de séjour nécessaire (Fig. 2a).

La collecte et l'évacuation des cendres et mâchefers sont assurées par des trémies placées en bas de la chambre et comportant des trappes de vidange.

L'oxydation des distillats de gaz nocifs provenant du four tournant est assurée par un brûleur à gaz et un apport d'air neuf, éventuellement préchauffé par un récupérateur de chaleur placé en aval de la postcombustion. L'agencement des entrées des différents flux de gaz est réalisé de sorte à assurer des conditions de turbulence favorables.

Le dimensionnement de cette chambre a été établi sur la base d'une approche globale simplifiée en utilisant des grandeurs moyennes au détriment des valeurs locales.
Les inconnues principales du problème sont les trois longueurs caractéristiques suivant les axes $x, y$ et $z$, ainsi que la puissance du brûleur. Les équations nécessaires à la résolution ont été obtenues en exprimant, dans un premier temps, une relation entre le débit total des gaz entrant dans la chambre et le temps de séjour. Une deuxième relation est donnée par la puissance du brûleur nécessaire à la mise en température du réfractaire pendant la phase transitoire et la surchauffe des gaz en régime permanent [1].

Deux critères qualitatifs ont été énoncés pour la définition complète de la géométrie. La première est relative à la qualité de l'écoulement des gaz alors que la seconde est associée à des conditions d'encombrement.

\section{Modélisation physique}

L'objectif de la modélisation est de pouvoir décrire les phénomènes physiques caractérisant l'écoulement turbulent réactif dans la chambre de postcombustion couplé avec les transferts thermiques par l'écriture des lois de conservation mettant en jeu plusieurs variables d'états.

On a considéré que les fumées, l'air neuf et les produits de combustion vérifient les hypothèses de gaz parfait, de fluide visqueux newtonien compressible et d'écoulement turbulent.

\section{1 Équations de l'aérothermie}

Le système est formé des équations de Navier-Stokes (continuité et quantités de mouvement), de l'énergie, et de l'équation d'état [6]. En appliquant l'opérateur des moyennes classiques et en utilisant la définition de la moyenne de Favre [7] aux équations de l'aérothermie, on 


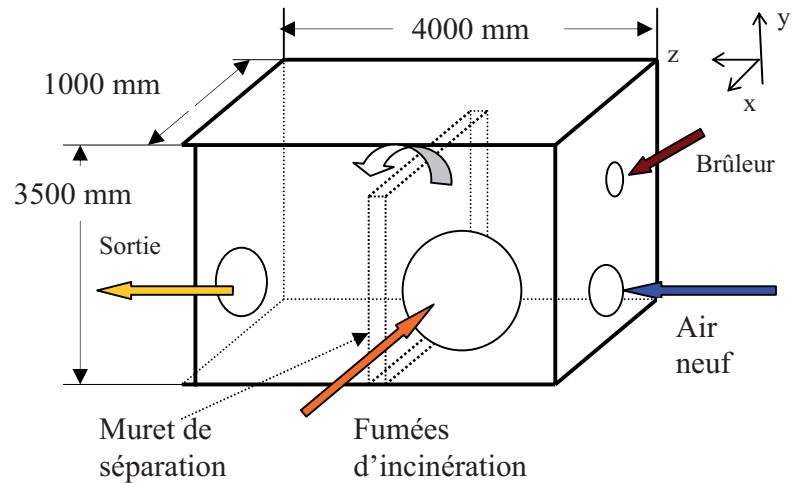

a) Géométrie.

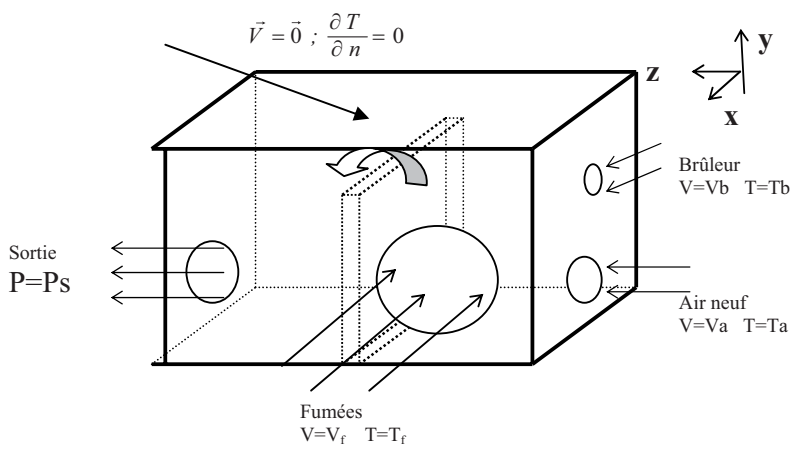

b) Conditions aux limites.

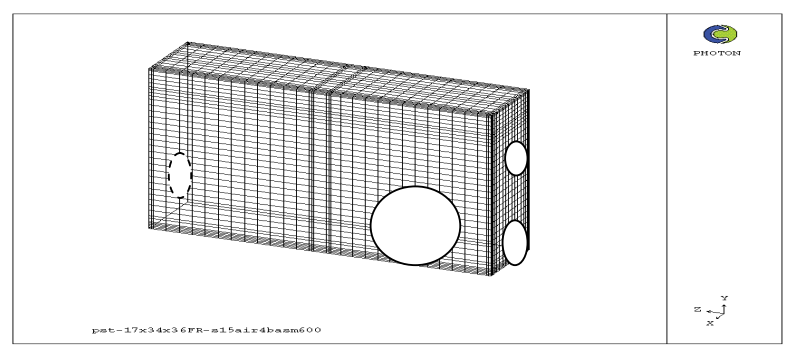

c) Maillage du domaine d'étude.

Fig. 2. Chambre de la postcombustion.

aboutit au système d'équations en valeurs moyennes suivant :

$$
\begin{aligned}
\frac{\partial}{\partial x_{i}}\left(\bar{\rho} \tilde{V}_{i}\right)= & 0 \\
\bar{\rho} \tilde{V}_{j} \frac{\partial \tilde{V}_{i}}{\partial x_{j}}= & -\frac{\partial \bar{P}}{\partial x_{i}}+\mu\left(\frac{\partial^{2} \bar{V}_{i}}{\partial x_{j} \partial x_{j}}+\frac{\partial^{2} \bar{V}_{j}}{\partial x_{j} \partial x_{i}}-\frac{2}{3} \frac{\partial^{2} \bar{V}_{j}}{\partial x_{i} \partial x_{j}}\right) \\
& -\frac{\partial \overline{\rho V_{i}^{\prime \prime}} V_{j}^{\prime \prime}}{\partial x_{j}}+\bar{f}_{i} \\
\bar{\rho} \tilde{V}_{j} \frac{\partial \tilde{h}}{\partial x_{j}}= & \frac{\partial}{\partial x_{j}}\left(\frac{\lambda}{C_{\mathrm{p}}} \frac{\partial \tilde{h}}{\partial x_{j}}-\overline{\rho V_{j}^{\prime \prime} h^{\prime \prime}}\right)+\bar{r}+\tilde{V}_{j} \frac{\partial \bar{P}}{\partial x_{j}} \\
& +\overline{V_{j}^{\prime \prime}} \frac{\partial \bar{P}}{\partial x_{j}}+\frac{\partial V_{j}^{\prime \prime}}{\partial x_{j}} \\
\bar{P}= & R \bar{\rho} \tilde{T}
\end{aligned}
$$

\subsection{Modélisation de la turbulence}

On adopte le modèle de turbulence classique $K-\varepsilon$, à deux équations de transport : l'équation de l'énergie cinétique turbulente $K$ et l'équation du taux de dissipation turbulente $\varepsilon[8,9]$. Ce modèle suppose l'hypothèse de Boussinesc sur le tenseur des contraintes turbulentes.

$$
\begin{aligned}
\bar{\rho} \tilde{V}_{j} \frac{\partial \tilde{K}}{\partial x_{j}} & =\frac{\partial}{\partial x_{j}}\left[\left(\mu+\mu_{\mathrm{t}}\right) \frac{\partial \tilde{K}}{\partial x_{j}}\right]+\operatorname{Prod}+P_{\mathrm{c}}-\bar{\rho} \varepsilon \\
\bar{\rho} \tilde{V}_{j} \frac{\partial \varepsilon}{\partial x_{j}} & =\frac{\partial}{\partial x_{j}}\left[\frac{\bar{\rho} \nu_{\mathrm{t}}}{\sigma_{\varepsilon}} \frac{\partial \varepsilon}{\partial x_{j}}\right]+\frac{\varepsilon}{K}\left[C_{\varepsilon 1}\left(\operatorname{Prod}+P_{\mathrm{c}}\right)-C_{\varepsilon 2} \bar{\rho} \varepsilon\right] \\
\operatorname{Prod} & =-\overline{\rho V_{i}^{\prime \prime} V_{j}^{\prime \prime}} \frac{\partial \tilde{V}_{i}}{\partial x_{j}}>0 \quad \text { et } \quad P_{\mathrm{c}}=-\frac{d_{\mathrm{t}}}{\bar{\rho}} \frac{\partial \bar{\rho}}{\partial x_{i}} \frac{\partial \bar{P}}{\partial x_{i}}
\end{aligned}
$$

La viscosité turbulente s'exprime en fonction de $\tilde{K}$ et $\varepsilon$ suivant l'hypothèse de Prandtl-Kolmogorov à l'aide de la constante empirique $C \approx 0,3$ :

$$
\nu_{\mathrm{t}}=C \frac{\tilde{k}^{2}}{\varepsilon}
$$

D'autre part, la longueur de mélange $l_{\mathrm{m}}$ s'exprime en fonction de $\tilde{K}$ et $\varepsilon$ :

$$
l_{\mathrm{m}}=\frac{\tilde{k}^{3 / 2}}{\varepsilon}
$$

Ce qui permet d'exprimer $\nu_{\mathrm{t}}$ sous la forme :

$$
\nu_{\mathrm{t}}=C \sqrt{\tilde{K}} l_{\mathrm{m}}
$$

\section{3 Équations des espèces réactives}

Les équations des fractions massiques des espèces réactives décrivent l'évolution d'un milieu à plusieurs espèces chimiques. Elles consistent à faire le bilan de la variation de chacune des variables dans un volume élémentaire. La variation dans le temps de la fraction massique $Y_{n}$ du constituant $n$, contenue dans un volume élémentaire est due à la convection, à la diffusion et à une réaction chimique. Ainsi, l'équation de transport de la fraction massique de l'élément $n$ en écoulement permanent s'écrit :

$$
\frac{\partial}{\partial x_{j}}\left(\rho Y_{n} V_{j}\right)=\frac{\partial}{\partial x_{j}}\left(-J_{i, j}\right)+\rho \omega_{n}
$$

avec :

$J_{i}$ : le flux de masse à travers une surface placée à l'abscisse $x_{i}$;

$\omega_{n}$ : taux de réaction chimique ou vitesse de consommation (ou de production) du constituant $n$ par une réaction chimique élémentaire.

Dans le cas d'un écoulement réactif turbulent, l'application de l'opérateur de moyenne arithmétique à 
l'équation (11) et l'utilisation des propriétés de la moyenne de Favre, conduisent à l'équation suivante des fractions massiques moyennes des espèces réactives :

$$
\underbrace{\frac{\partial}{\partial x_{j}}\left(\bar{\rho} \tilde{Y}_{n} \tilde{V}_{j}\right)}_{\mathrm{I}}=\underbrace{\frac{\partial}{\partial x_{j}}\left(-\bar{J}_{i, j}\right)}_{\mathrm{II}}+\underbrace{\bar{\rho} \tilde{\omega}_{n}}_{\mathrm{III}}-\underbrace{\frac{\partial}{\partial x_{j}}\left(\overline{\rho Y_{n}^{\prime \prime} V_{j}^{\prime \prime}}\right)}_{\mathrm{IV}}
$$

L'utilisation de cette équation pose le problème de la modélisation du terme de diffusion turbulente (terme IV) et du taux moyen de réaction $\tilde{\omega}_{n}$.

\section{Hypothèses :}

- On suppose que les mécanismes réactionnels sont rapides de telle sorte que les phénomènes turbulents l'emportent sur les réactions chimiques. Ainsi, on néglige le flux de diffusion moléculaire (terme II) devant celui de la diffusion turbulente de masse (terme IV).

- La diffusion turbulente de masse est supposée proportionnelle au flux de masse en introduisant un coefficient de diffusion turbulente de masse $d_{\mathrm{t}}$ :

$$
\overline{\rho Y_{n}^{\prime \prime} V_{j}^{\prime \prime}}=\bar{\rho} d_{\mathrm{t}} \frac{\partial \tilde{Y}_{n}}{\partial x_{j}}
$$

avec :

$$
d_{\mathrm{t}}=\beta L_{\mathrm{t}} \sqrt{\tilde{K}}
$$

$\beta$ est une constante et $L_{\mathrm{t}}$ est la taille moyenne des tourbillons.

- Modèle E.B.U. «Eddy Break Up» pour le taux de réaction moyen $\tilde{\omega}_{i}[10,11]$ :

ce modèle, proposé dans le cas des flammes turbulentes de pré-mélange, est basé sur les hypothèses suivantes :

- On suppose que les phénomènes chimiques sont très rapides devant les phénomènes d'écoulement turbulent [10-12]. Ainsi, les mécanismes réactionnels sont négligeables devant les phénomènes turbulents.

- On adopte l'hypothèse de l'état quasi-stationnaire : on considère le bilan d'une réaction globale à l'équilibre chimique en supposant que le taux de transformation des réactifs est égal au taux de production des produits. Cette hypothèse a un domaine de validité très large et simplifie considérablement les calculs.

Le modèle EBU introduit le taux moyen de réaction $\tilde{\omega}_{n}$ en fonction d'une variable $C_{n}$ qui représente le degré d'avancement de la combustion (progress variable) :

$$
\begin{aligned}
\tilde{\omega}_{n} & =-C_{\mathrm{EBU}} \frac{\tilde{C}_{n}\left(1-\tilde{C}_{n}\right)}{\tau_{t}}\left(Y_{n}^{0}-Y_{n}^{\mathrm{eq}}\right) \\
C_{n} & =\frac{Y_{n}^{0}-Y_{n}}{Y_{n}^{0}-Y_{n}^{\mathrm{eq}}} \quad 0 \leqslant C_{n} \leqslant 1 \\
\tau_{t} & =\frac{l_{t}}{\tilde{k}^{1 / 2}}
\end{aligned}
$$

avec :

$C_{\mathrm{EBU}}$ : constante empirique du modèle Eddy Break Up; $C_{n}$ : variable du degré d'avancementde la réaction, elle varie de 0 à 1 entre les gaz frais et brûlés;

$\tau_{\mathrm{t}}$ : échelle de temps des grands tourbillons;

$Y_{n}^{0}$ : proportion du gaz $n$ avant réaction;

$Y_{n}^{\text {eq }}$ : proportion du gaz $n$ à l'équilibre chimique ;

$l_{\mathrm{t}}$ : longueur caractéristique de la turbulence.

Ainsi, l'équation (12) des fractions massiques moyennes se réduit à l'équation suivante dont l'inconnue devient $\tilde{C}$ :

$$
\frac{\partial}{\partial x_{j}}\left(\bar{\rho} \tilde{C}_{n} \tilde{V}_{j}\right)=\frac{\partial}{\partial x_{j}}\left(\bar{\rho} d_{\mathrm{t}} \frac{\partial \tilde{C}_{n}}{\partial x_{j}}\right)+C_{\mathrm{EBU}} \frac{\tilde{C}_{n}\left(1-\tilde{C}_{n}\right)}{\tau_{t}}
$$

Ce modèle, couplé avec le modèle de turbulence $K-\varepsilon$, nous est apparu suffisant dans l'optique développée ici et les objectifs envisagés par cette étude.

\section{Conditions aux limites}

Le domaine fluide considéré comporte trois entrées (fumées à incinérer, air neuf et gaz brûleur) et une sortie (Fig. 2b).

Les parois solides qui délimitent le domaine sont traitées avec des conditions cinématiques d'adhérence $(\boldsymbol{V}=0)$ et sont considérées adiabatiques : $\frac{\partial T}{\partial n}=0$.

Aux entrées, on impose une vitesse égale à la vitesse débitante et une température d'entrée. À la sortie, on impose la pression.

Les fumées d'incinération pénètrent dans la chambre de postcombustion à travers une section circulaire de diamètre 1,8 m (Fig. 1). Le débit nominal des fumées générées par le four tunnel est de $5420 \mathrm{~m}^{3} \cdot \mathrm{h}^{-1}$ à $800 \mathrm{~K}$ et 1 bar.

L'enthalpie massique des fumées à l'entrée est calculée par la relation $h_{\mathrm{f}}=C_{\mathrm{pf}} \cdot T_{\mathrm{f}}+P C I_{\mathrm{c}} \cdot Y_{\mathrm{c}}$ en considérant le pouvoir calorifique inférieur des combustibles présents dans les fumées $P C I_{\mathrm{c}}$ et $Y_{\mathrm{c}}$ leur fraction massique. En réalité, une grande difficulté existe à ce niveau concernant la composition des gaz émis par le four très variable en fonction de la nature aléatoire des déchets incinérés. En première estimation, on a considéré une proportion de gaz combustible $Y_{\mathrm{c}}$ de $2 \%$, de pouvoir calorifique moyen

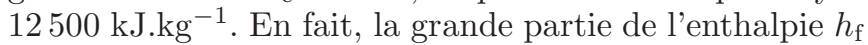
est constituée par la chaleur sensible due à la température $T_{\mathrm{f}}$ des fumées.

Le brûleur génère une flamme turbulente de prémélange (gaz naturel/air). Le combustible ainsi que le comburant alimentent le brûleur à la température ambiante $T_{\mathrm{b}}$ et à la pression atmosphérique. Le débit des gaz est calculé à partir de la puissance nominale du brûleur (1,5 MW), du pouvoir fumigène du combustible $\left(10,57 \mathrm{Nm}^{3}\right.$ fumées $/ \mathrm{Nm}^{3}$ gaz) et d'un excès d'air de $20 \%$, le PCI du combustible étant de $39700 \mathrm{~kJ} . \mathrm{m}^{-3}$.

L'air neuf, préchauffé à travers un récupérateur, est admis à $480 \mathrm{~K}$ et à la pression atmosphérique avec un débit de $3140 \mathrm{~m}^{3} \cdot \mathrm{h}^{-1}$. 
L'utilisation d'un extracteur de fumées impose au niveau de la section de sortie de la chambre de postcombustion une dépression $P_{\underline{s}}$ de $-200 \mathrm{~Pa}$.

Concernant les grandeurs d'entrée relatives à la turbulence, l'énergie cinétique turbulente moyenne $\tilde{K}$ au niveau des différentes entrées, relativement faible, est estimée égale à $2 \%$ de l'énergie cinétique de l'écoulement moyen. L'énergie moyenne de dissipation visqueuse $\varepsilon$ est calculée en utilisant l'hypothèse de Prandtl-Kolmogorov suivant l'équation (9). La longueur de mélange $l_{\mathrm{m}}$ est estimée égale à $1 / 10$ du diamètre de la section d'entrée.

\section{Approximations numériques}

\subsection{Discrétisation géométrique}

Une approche réaliste du problème posé, caractérisé par un mélange d'écoulements croisés, nécessite l'adoption d'un domaine de calcul tridimensionnel en dépit de l'augmentation très sensible de la taille des calculs que cela implique. Relativement à la méthode des volumes finis retenue pour cette étude, nous avons adopté un maillage tridimensionnel structuré du domaine fluide (Fig. 2c). Il est bien évident que la qualité des résultats dépendra directement de la finesse de ce maillage. Dans cette optique, et pour évaluer la sensibilité des paramètres calculés au maillage, nous avons augmenté progressivement le nombre de nœuds dans les trois directions jusqu'à l'obtention d'une solution relativement stable avec un temps de calcul raisonnable. Le maillage final adopté comporte 19 nœuds suivant $x, 40$ nœuds suivant $y$ et $z$ soit un total de 32400 nœuds.

\subsection{Discrétisation des équations}

Les calculs sont effectués à l'aide du code Phoenics [5]. Celui-ci permet de traiter les équations précédentes de transport de l'aéro-thermo-chimie qui prennent la forme générale suivante pour chaque variable $\Phi$ :

$$
\frac{\partial \rho \Phi}{\partial t}+\operatorname{div}(\rho \Phi \boldsymbol{V})=\operatorname{div}\left(\Gamma_{\Phi} \operatorname{grad} \Phi\right)+S_{\Phi}
$$

où $\Gamma_{\Phi}$ représente le coefficient de diffusion et $S_{\Phi}$ le terme source. La résolution de ce système non-linéaire d'équations aux dérivées partielles est basée sur la formulation d'une équation algébrique de chaque variable en tout point $\mathrm{P}$ obtenue à partir de la discrétisation et l'intégration de l'équation différentielle correspondante sur chacun des volumes de contrôle créés par le maillage [13-15]. La méthode de résolution itérative adoptée pour la détermination de la pression est basée sur l'algorithme Simplest [14], basé sur l'incrémentation itérative d'un champ de pression jusqu'à la vérification de l'équation de l'équilibre pour un régime permanent. Les termes de convection et de diffusion sont approchés par un schéma de différences centrées du second ordre [1315]. Les composantes de la vitesse sont calculées aux

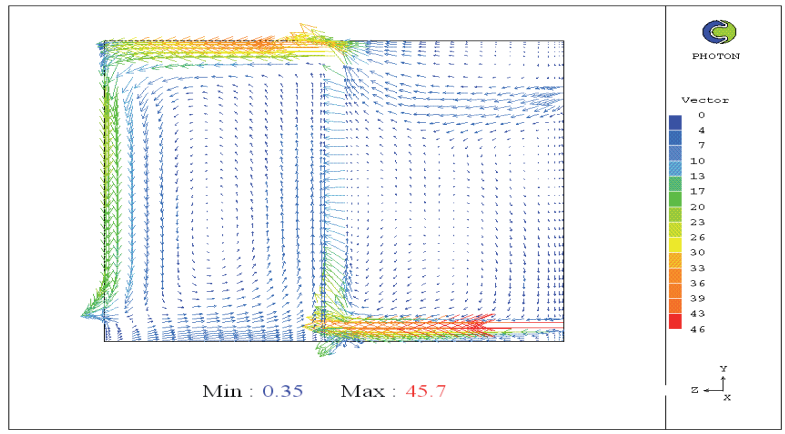

Fig. 3. Vecteurs vitesses (ouverture : $150 \mathrm{~mm}$ ).

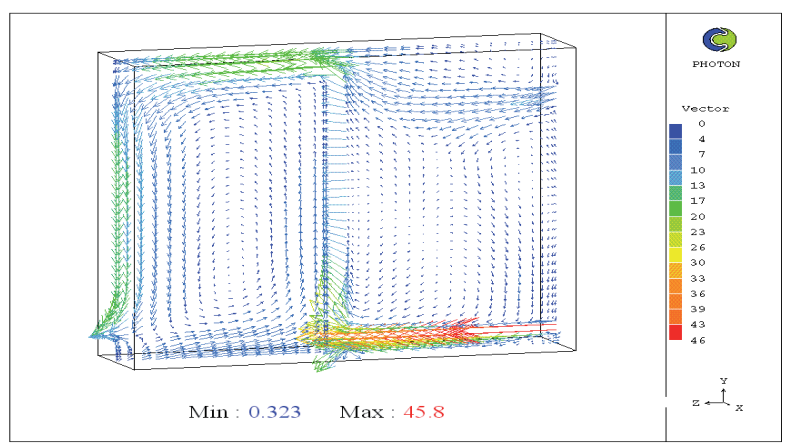

Fig. 4. Vecteurs vitesses (ouverture : $450 \mathrm{~mm}$ ).

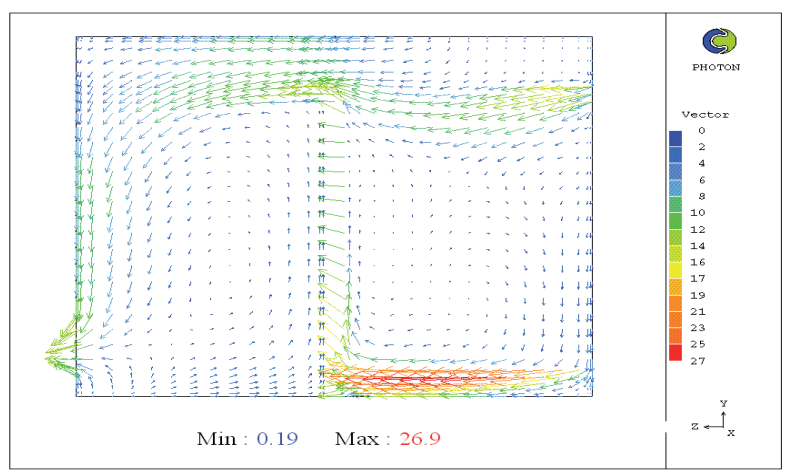

Fig. 5. Vecteurs vitesses (ouverture : $650 \mathrm{~mm}$ ).

centres des faces des cellules et les autres variables sont déterminées aux centres des cellules. La stabilité du processus itératif est assurée par l'emploi d'un coefficient de sous-relaxation linéaire. La convergence de la méthode est contrôlée par l'examen de l'évolution de l'ensemble des variables en un point choisi et des résidus relatifs à chacune des équations correspondantes en fonction du nombre d'itérations. La qualité de la convergence se traduit par l'invariance de ces paramètres.

Ainsi, le problème traité met en jeu 11 variables :

- 3 composantes de la vitesse : $V_{1}, V_{2}$ et $V_{3}$;

- 3 variables d'état : pression $P$, température $T$ et masse volumique $\rho$;

- 2 grandeurs turbulentes : énergie cinétique $K$ et taux de dissipation $\varepsilon$;

- 3 fractions massiques des espèces réactives : $Y_{\text {air }}$, $Y_{\text {combustible }}$ et $Y_{\text {produits }}$. 


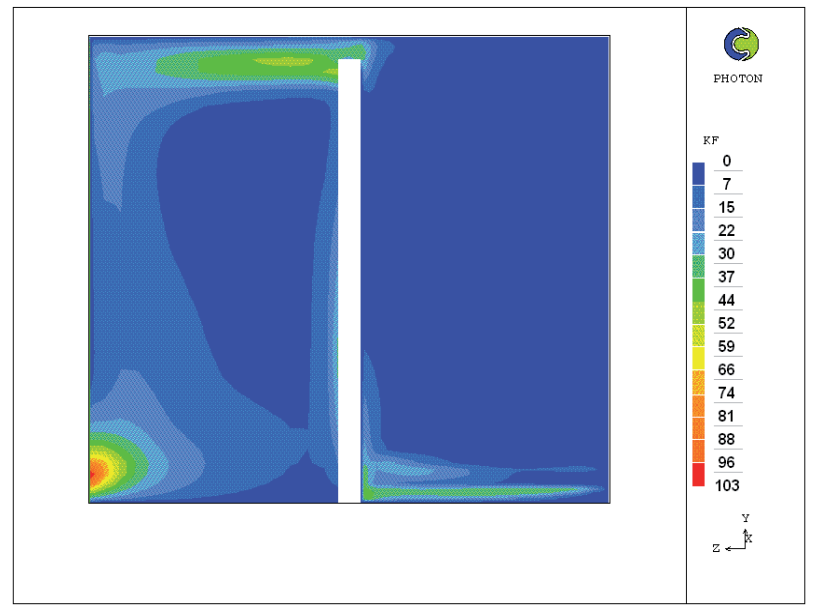

Ouverture : $150 \mathrm{~mm}$.

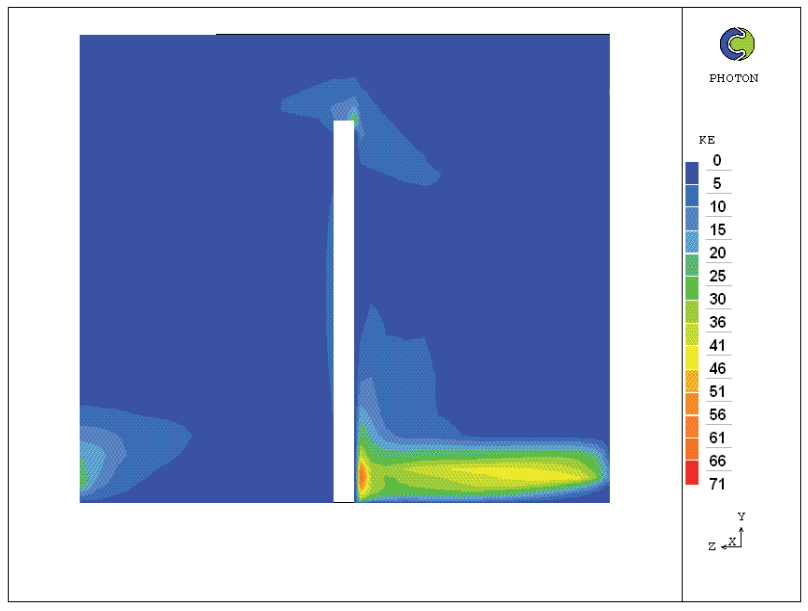

Ouverture : $650 \mathrm{~mm}$.

Fig. 6. Énergie cinétique turbulente KE.

\section{Paramètres de simulation}

Nous avons sélectionné, pour les simulations, les paramètres accessibles au stade de la réalisation de l'installation et qui ont une grande influence sur le comportement aérothermique de la chambre, soit :

- la variation de l'ouverture du muret de séparation des deux compartiments ;

- la variation du nombre de gicleurs d'air neuf;

- la variation de la position des gicleurs.

\section{Résultats et discussions}

Les résultats des simulations sont analysés suivant le plan milieu de la chambre $(x=0,5 \mathrm{~m})$.

\subsection{Influence de l'ouverture du muret de séparation}

Nous avons effectué des simulations pour trois valeurs des ouvertures du muret de séparation : $150 \mathrm{~mm}, 450 \mathrm{~mm}$ et $650 \mathrm{~mm}$.

Les figures 3-5 montrent bien la structure des écoulements de mélange qui s'établissent dans la première chambre et l'apparition d'une large zone tourbillonnaire de sens anti-horaire centrée sur le second compartiment.

Il apparaît ainsi que la disposition adoptée avec une inclinaison du brûleur favorise convenablement le mélange des fumées, air neuf et gaz brûleur dans le premier compartiment et que le deuxième compartiment constitue un réacteur avec un mouvement tourbillonnaire de l'ensemble en légère dépression, ce qui justifie le choix de cette disposition géométrique.

On remarque également que les vitesses de passages à travers l'ouverture au-dessus du muret de séparation sont élevées (environ $40 \mathrm{~m} . \mathrm{s}^{-1}$ ) dans le cas de la plus petite ouverture $(150 \mathrm{~mm})$ ce qui peut provoquer des zones d'érosion. Par ailleurs, le temps de séjour des fumées dans ce cas, estimé à partir d'une intégration le long des lignes de courant, est limité à $0,7 \mathrm{~s}$.

Le cas d'un muret d'ouverture $650 \mathrm{~mm}$, correspond au temps de séjour le plus élevé soit environ 1,7 s.

L'énergie cinétique turbulente maximale est localisée aux niveaux des singularités qui accélèrent l'écoulement : entrée d'air neuf, ouverture au-dessus du muret et sortie (Fig. 6). Ailleurs et dans le reste du domaine où les vitesses sont plus petites, elle devient très faible. Pour l'ensemble des cas, elle a une valeur moyenne de répartition dans la chambre de l'ordre de $15 \mathrm{~m}^{2} . \mathrm{s}^{-2}$ ce qui ne reflète pas une turbulence importante. Ce résultat est dû en grande partie au volume important de la chambre. Par ailleurs, la dissipation de l'énergie turbulente $\tilde{\varepsilon}$ a une valeur de répartition presque homogène égale à $167 \mathrm{~m}^{2} \cdot \mathrm{s}^{-3}$ à part la zone d'injection d'air neuf.

La figure 7 représente le champ des températures pour les trois ouvertures. Il apparaît clairement que le brûleur génère une flamme assez large avec des températures locales qui dépassent $2000{ }^{\circ} \mathrm{C}$. Le mélange des produits de cette flamme avec l'air neuf et les fumées du four conduit dans le second compartiment à une température relativement homogène qui reste, dans tous les cas, supérieure à $1000{ }^{\circ} \mathrm{C}$ ce qui est nécessaire pour l'oxydation des gaz. Ceci confirme bien le rôle de réacteur thermique joué par le deuxième compartiment. Pour la petite ouverture (150 mm), les gaz chauds ont tendance à lécher la paroi supérieure du four ce qui peut accentuer les fuites thermiques. L'augmentation de cette ouverture conduit à une répartition plus homogène des températures en épargnant cette paroi.

La figure 8 montre la répartition des espèces réactives. On constate tout naturellement que le taux d'air est élevé dans le premier compartiment alors que le taux des produits de combustion est élevé au niveau du brûleur et dans le deuxième compartiment. 


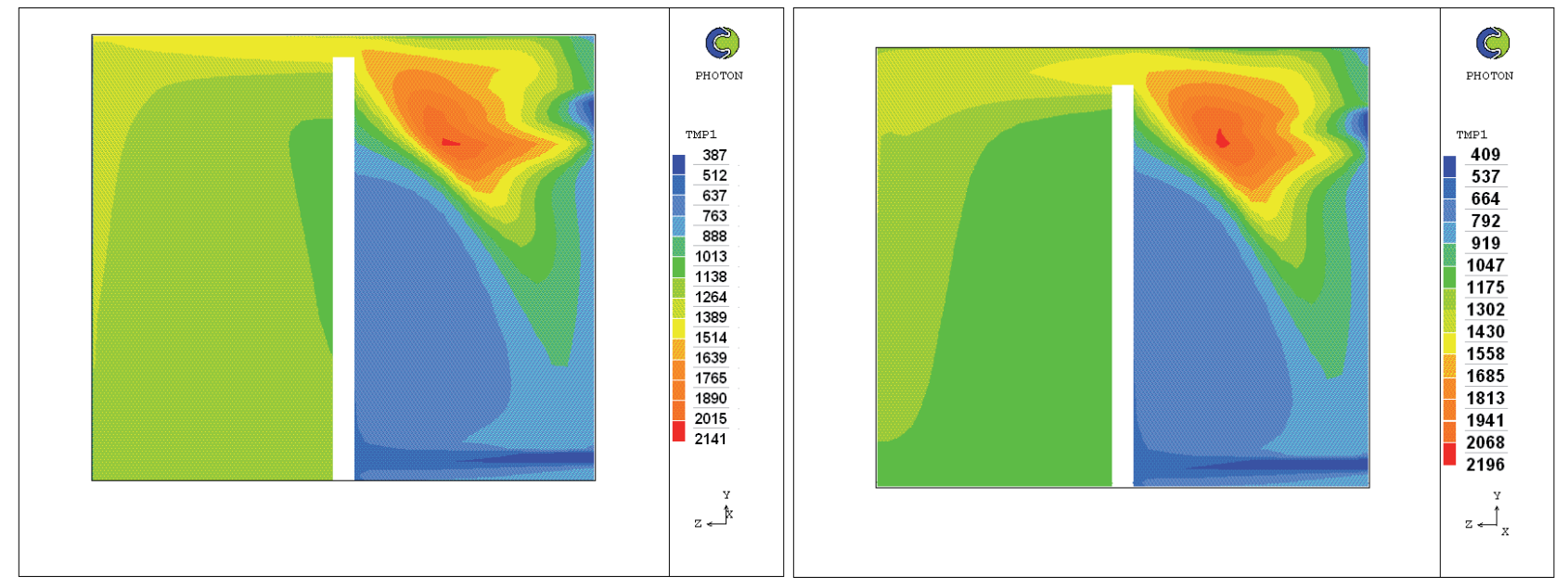

Ouverture : $150 \mathrm{~mm}$.

Ouverture : $450 \mathrm{~mm}$.

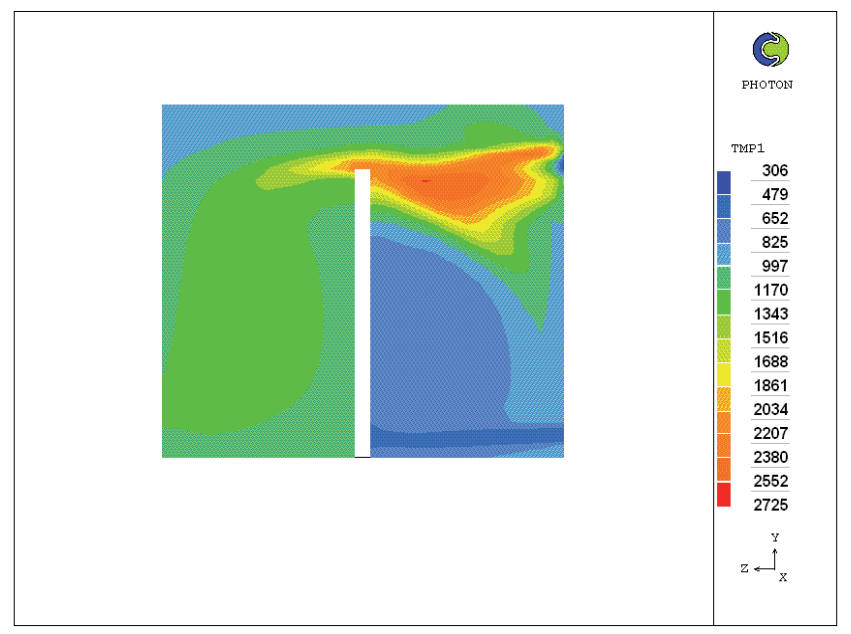

Ouverture : $650 \mathrm{~mm}$.

Fig. 7. Champ de température.

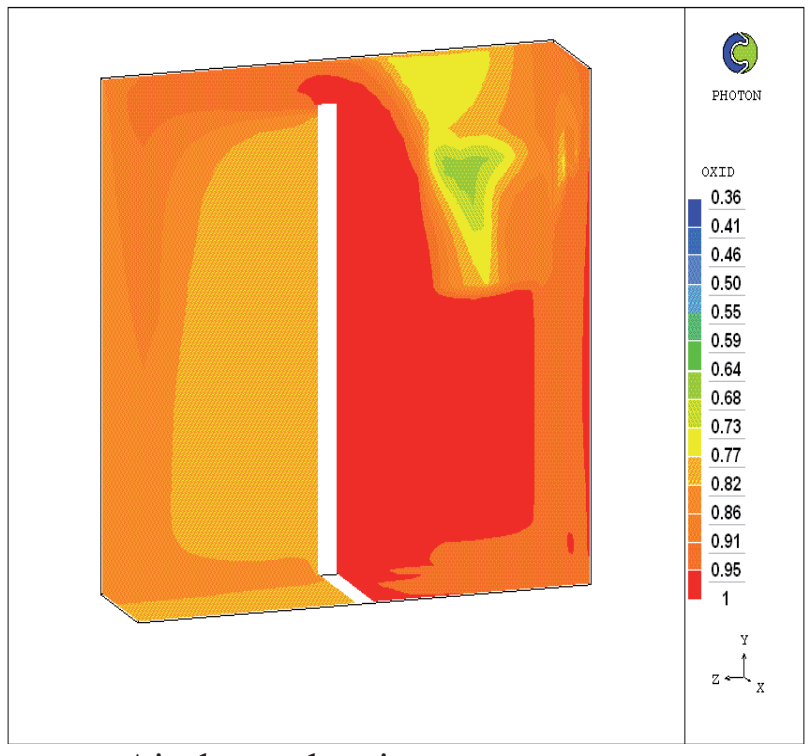

Air de combustion.

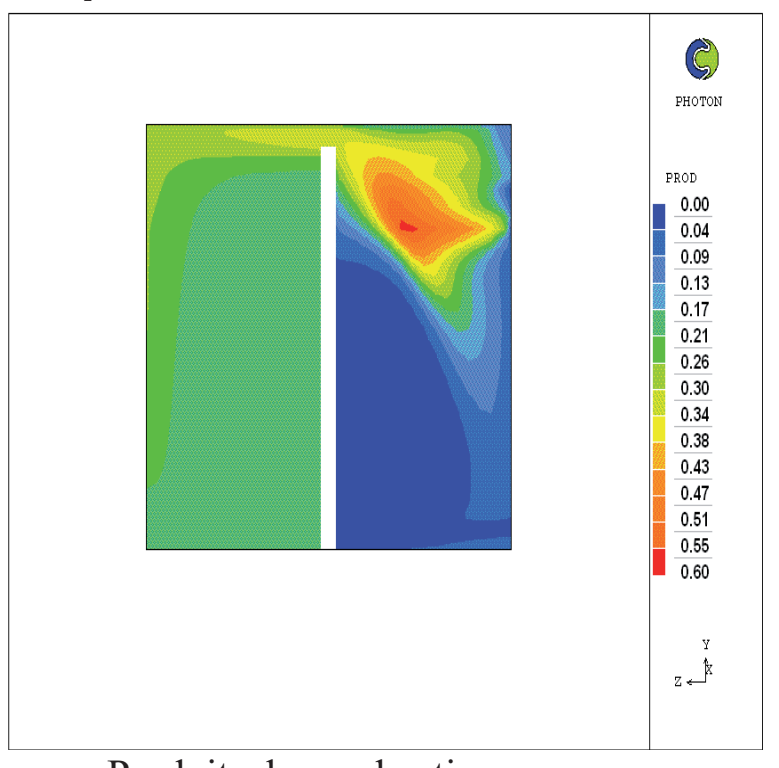

Produits de combustion.

Fig. 8. Répartition des fractions massiques. 


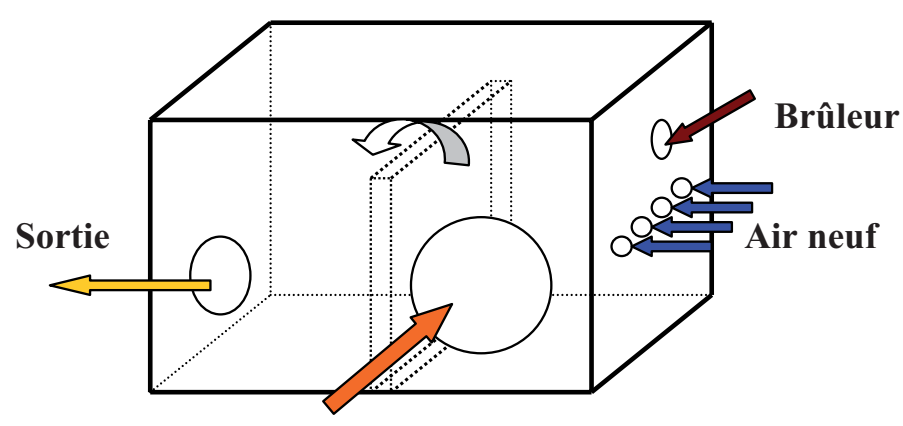

Fumées

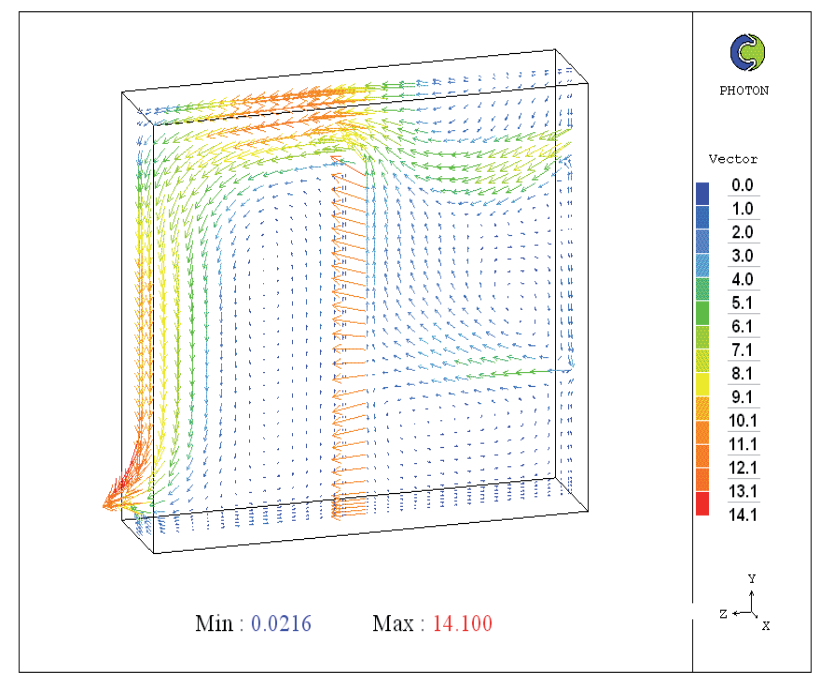

a) Champ des vitesses.
Fig. 9. Disposition de la chambre avec des injecteurs d'air en position milieu.

Fig. 10. Injecteurs d'air en position milieu : a) champ des vitesses ; b) champ de température.

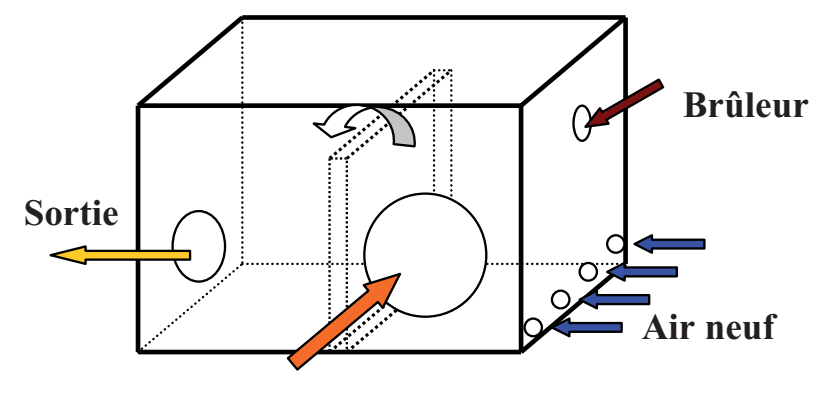

Fumée

Fig. 11. Injecteurs d'air neuf en position basse.

\subsection{Influence du nombre d'injecteurs d'air neuf et de leurs positions}

On a remplacé la source d'alimentation en air neuf par quatre sources à débit total égal. Quant à la position des sections d'entrée des injecteurs, on a considéré en premier lieu qu'ils sont à la même hauteur que celle des fumées à incinérer (Fig. 9). Cette disposition est envisagée dans le but d'accentuer le mélange des fumées provenant du four avec l'air neuf et la diffusion de la flamme du brûleur dont l'axe est toujours légèrement incliné vers le bas.
La figure 10a montre l'apparition de part et d'autre des jets d'air de deux zones tourbillonnaires de sens contraire. Le tourbillon supérieur serait favorable au mélange avec les gaz du brûleur alors que celui du bas peut favoriser localement l'installation d'une zone morte. Le temps de séjour dans ce cas augmente jusqu'à environ $0,9 \mathrm{~s}$ pour une ouverture de $150 \mathrm{~mm}$. Au niveau des grandeurs turbulentes, on n'a pas observé de différences significatives avec le cas d'une seule entrée.

On constate également sur la figure 10b que la flamme du brûleur est fortement déviée vers le haut entraînant une augmentation sensible de la température locale ce qui peut causer un endommagement de la paroi supérieure de la chambre.

Afin d'éviter les inconvénients de la disposition précédente, et pour atteindre le temps de séjour requis, on a envisagé une disposition des injecteurs d'air neuf dans la position basse (Fig. 11).

La structure de l'écoulement obtenu (Figs. 12a et b) montre bien la disparition de la zone morte alors que la taille du tourbillon au-dessus des jets augmente. Les vitesses d'écoulement sont plus faibles, le temps de séjour minimal dans ce cas est passé à $6 \mathrm{~s}$ pour le cas d'une ouverture de $650 \mathrm{~mm}$ ce qui est nettement meilleur que les cas précédents. La figure 13 montre que dans ce cas 


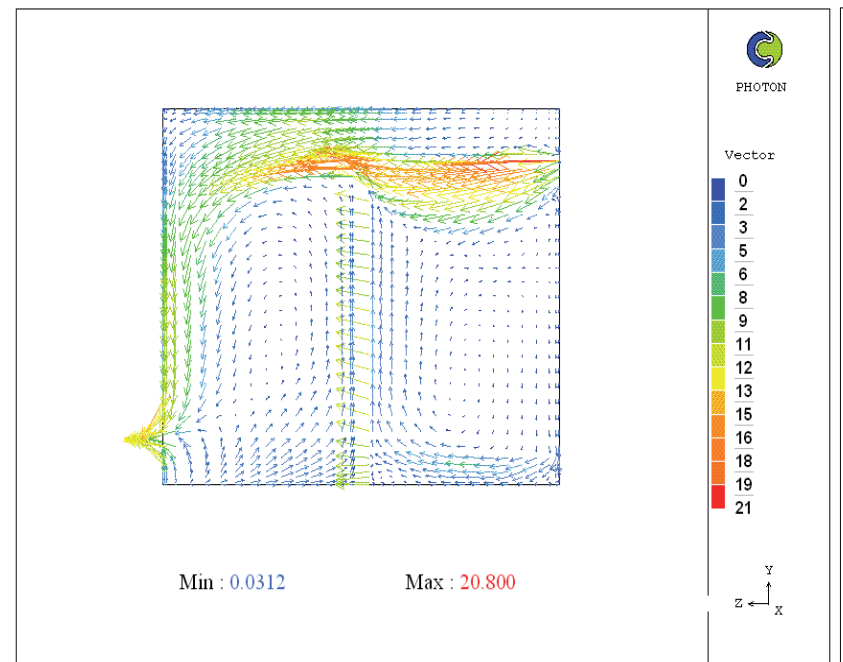

a) Champ des vitesses

Fig. 12. Injecteurs d'air neuf en position basse : a) champ des vitesses ; b) champ de courant.

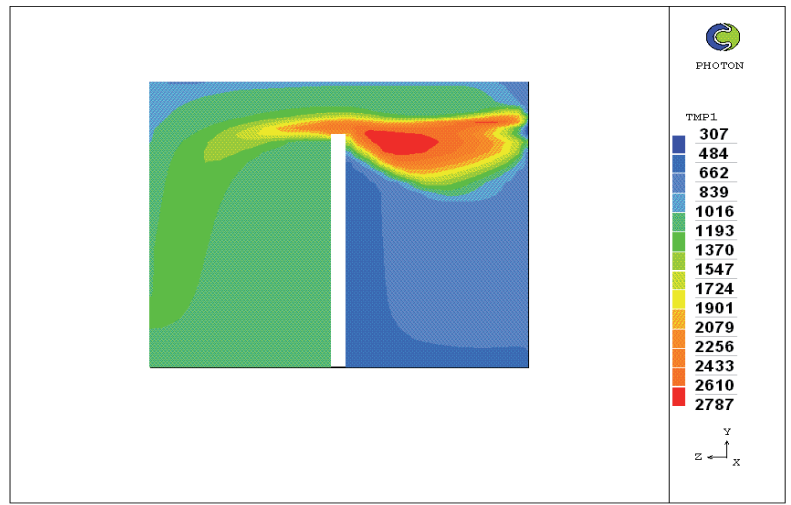

Fig. 13. Champ de température (injecteurs en position basse).

la température de la flamme et des gaz au deuxième compartiment augmente sensiblement. En effet, l'abaissement de la position d'alimentation en air neuf modifie la répartition du comburant dans le premier compartiment et diminue les effets de l'excès d'air neuf au niveau du brûleur dont la température locale de la flamme augmente ainsi jusqu'à $2700{ }^{\circ} \mathrm{C}$.

\section{Conclusion}

Ce travail a permis de développer par le biais d'un outil numérique un modèle de simulation de la chambre de postcombustion d'une installation d'incinération.

L'approche adoptée pour l'analyse des écoulements réactifs par le biais du modèle de combustion E.B.U. nous est apparue suffisante pour appréhender les processus physiques mis en jeu dans le cadre de la vision ingénierie qui sous-tend ce travail.

L'étude des cas correspondant aux différentes configurations des paramètres adoptés a permis d'une part de dégager l'influence de ces paramètres sur le respect de la règle des trois $\mathrm{T}$, et d'autre part de discuter la validité de la conception adoptée.

On constate que la température dans toute la chambre dans la configuration actuelle est au-dessus de la valeur minimale exigée.

L'écoulement reste cependant modéré en ce qui concerne la turbulence, ce qui suggère l'introduction de dispositions complémentaires lors de la réalisation du garnissage réfractaire. En effet, la turbulence peut être accentuée par l'adjonction de déflecteurs internes au niveau du premier compartiment de la chambre.

Quant au temps de séjour, la simulation avec quatre sources d'air neuf disposées en bas de la chambre, avec une ouverture du muret de $650 \mathrm{~mm}$, montre en analysant les vecteurs vitesses suivant les lignes de courant que le temps de séjour minimal de deux secondes peut être assuré si la conception réelle est améliorée par la réalisation d'entrées d'air multiples dans le garnissage réfractaire même si l'entrée principale reste unique.

Les essais expérimentaux qui pourraient accompagner l'étude de ce type de projets industriels nécessitent la mise en place de prototypes et d'une instrumentation dont les délais de réalisation sont longs et les coûts prohibitifs. Une simulation opérationnelle et réaliste permet de réduire les délais et d'économiser des essais. Cette étude peut s'intégrer dans le cadre de cette nouvelle approche que certains appellent la Simulation Based Design ou SBD (le dimensionnement basé sur la simulation).

\section{Références}

[1] M. Jemmali, J. Bessrour, A. Ben Rhima, M. Bouhafs, Incinérateur de déchets septiques hospitaliers, rapport interne, ENIT 2002

[2] F. Marias, J.-R. Puiggali, Simulation numérique d'un brûleur industriel, IJTS 39 (2000) 249-264 
[3] J.P. Chabard, P.L. Viollet, Les défis des codes de mécanique des fluides pour les années à venir, Revue générale de thermique 356-357 (1991) 553-561

[4] B. Poirault, Mécanisme de combustion dans un brûleur méthane-air, influence de l'intensité de rotation, thèse université de Poitiers, 1997

[5] H.I. Rosten, D.B. Spalding, Manuel d'utilisation du code PHOENICS, CHAM, 1999

[6] J.F. Sacadura, Transfert thermique, Techniques et documentation, Paris, 1980

[7] P. Viollet, Mécanique des fluides à masse volumique variable, Presse des Ponts et Chaussées, 1997

[8] R. Scheistel, Modélisation et simulation des écoulements turbulents, Ed. Hermès, Paris, 1993

[9] P. Bradshaw, Turbulence, Springer-Verlag, 1976
[10] R. Borghi, M. Destriau, La combustion et les flammes, Ed. Technip, 1995

[11] R. Borghi, M. Champion, Modélisation et théorie des flammes, Ed. Technip, 2000

[12] R. Borghi, La modélisation de la combustion turbulente non pré mélangée : un problème déjà résolu?, Revue scientifique et technique de la défense 1 (1994)

[13] H. K. Verstag, W. Malalasekera, An introduction to computational fluid dynamic, The finite volume method, Longman Group Ltd, 1995

[14] S.V. Patankar, Numerical heat transfer and fluid flow, Hemisphere Publishing Corporation, 1980

[15] Z.U.A. Warsi, Fluid Dynamics: Theoretical and Computational Approches, CRC, 1999 